\title{
Possitively charged ferritin nanocages can deliver siRNA into cells
}

\author{
Marketa Charousova1,2, Michal Mokry ${ }^{3}$, Zbynek Splichal ${ }^{1,2}$, Simona Rex ${ }^{1,2}$, Vladimir Pekarik ${ }^{4}$, Zbynek \\ Heger $^{1,2}$ \\ ${ }^{1}$ Department of Chemistry and Biochemistry, Mendel University in Brno \\ Zemedelska 1, CZ-613 00 Brno, Czech Republic \\ ${ }^{2}$ Central European Institute of Technology, Brno University of Technology \\ Purkynova 656/123, CZ-612 00 Brno, Czech Republic \\ charousovam@gmail.com; zbynek.splichal@seznam.cz; simona.rex@mendelu.cz; zbynek.heger@mendelu.cz \\ ${ }^{3}$ Department of Biomedical Engineering, Brno University of Technology \\ Technicka 12, CZ-616 00 Brno, Czech Republic \\ xmokry10@vutbr.cz \\ ${ }^{4}$ Department of Physiology, Masaryk University \\ Kamenice 753/5, CZ-625 00 Brno, Czech Republic \\ pekarikv@mail.muni.cz
}

\section{Extended Abstract}

Nanomedicine is based on using nanotransporters for transfer of drugs and other active compounds inside cancer tissue without damaging the normal cells and organs [1]. For this purpose, various types of molecules can be used. Using proteins as nanocarriers is tested for their biocompatibility, safety and non-toxicity [2]. Interested protein based nanotransporter is ferritin. It is ubiquitous, with convenient size, robust, stable at various conditions and it is easy to modify its surface to achieve active targeting [3]. However, its best quality is the ability to open the protein cage in $\mathrm{pH}$ below 3 and folding it back together in $\mathrm{pH}$ above 7 [4]. This property is used for active encapsulation of various molecules. By mixing ferritin with the compounds of interest, lowering $\mathrm{pH}$ with $\mathrm{HCl}$ and then returning $\mathrm{pH}$ back to neutral by addition of $\mathrm{NaOH}$, we can encapsulated cargo inside ferritin cage [5].

Recombinant human ferritin (HsaFtH-RK) was designed and cloned under supervision of Dr. Pekarik. The production of this ferritin is done in bacterial strand E. coli - RIL. The structure of this human ferritin is spherical, consisting of 24 heavy subunits with outer diameter $12.6 \mathrm{~nm}$, and inner hollow cavity with diameter $8 \mathrm{~nm}$. This produced human ferritin is stable at high temperatures above $80^{\circ} \mathrm{C}$ and in $\mathrm{pH} 4-12$. It is possible to encapsulate various molecules by two ways: passive encapsulation - the solution of ferritin and wanted cargo is left standing for 2 and more hours depending on cargo size and charge; of active encapsulation - opening the ferritin structure by increasing the $\mathrm{pH}$ above 12 or lowering $\mathrm{pH}$ under 4 (this looses the structure of ferritin and cargo is able to get inside ferritin), followed by getting $\mathrm{pH}$ back to $7-8$ (the structure will spontaneously reassemble. For siRNA encapsulation, both passive and active encapsulation were tried. Passive encapsulation (overnight) was discarded, as we were not able to detect any encapsulated siRNA. Alkaline encapsulation protocol was chosen for active encapsulation. Specific amounts of $1 \mathrm{M} \mathrm{NaOH}$ and $1 \mathrm{M} \mathrm{HCl}$ had to be optimized. The final ration of $\mathrm{NaOH}$ and $\mathrm{HCl}$ was determined 1:1.13 for the best encapsulation efficiency. Fluorescently labelled siRNA (FAM-siRNA) was used in final concentration of $1 \mu \mathrm{M}$. Verification of correct encapsulation was done by agarose and native page with findings that we are able to encapsulate FAM-siRNA inside HsaFtH-RK and that HsaFtH-RK after encapsulation protocol is in its native structure with no aggregates. We were also able to verify that HsaFtH-RK is able to internalize inside cell lines, the pictures were taken after 12 hours of incubation. The ferritin was labelled with Cy5 fluorescence dye for visualization. The pictures taken on fluorescence microscope were used to localize ferritin inside lysosomes and also distributed in cell cytoplasm. Flow cytometry after 12 hours of incubation proved the internalization of HsaFtH-RK-FAM-siRNA into $25 \%$ of cells. Therefore, we are able to say that we created a stable nanoconstruct for siRNA cell delivery. 


\section{References}

[1] Todd, T.J. et al. "Ferritin nanocages: great potential as clinically translatable drug delivery vehicles?" Nanomedicine, 2013, 8(10), pp.1555-1557.

[2] Uchida, M. et al. "Targeting of cancer cells with ferrimagnetic ferritin cage nanoparticles." Journal of the American Chemical Society, 2006, 128(51), pp. 16626-16633.

[3] Chen, G. et al. "Apoferritin as a bionanomaterial to facilitate the electron transfer reactivity of hemoglobin and the catalytic activity towards hydrogen peroxide." Bioelectrochemistry, 2008, 72(1), pp. 77-80.

[4] Banyard, S.H. et al. "Electron density map of apoferritin at 2.8-Å resolution." Nature, 1978, 271(5642), pp. 282.

[5] Heger, Z. et al. “Apoferritin applications in nanomedicine.” Nanomedicine, 2014, 9(14), pp. 2233-2245. 